Virginia Commonwealth University vCU Scholars Compass

2014

\title{
A novel two-laser interface for coupling capillary electrochromatography with ion-trap time-of-flight mass spectrometry
}

David C. Simpson

Virginia Commonwealth University, dsimpson3@vcu.edu

Alexander J. Yates

University of Edinburgh

John H. Knox

University of Edinburgh

Pat R.R. Langridge-Smith

University of Edinburgh

Follow this and additional works at: http://scholarscompass.vcu.edu/chem_pubs

Part of the Chemistry Commons

Copyright (C) 2014 Elsevier B.V. NOTICE: This is the author's version of a work that was accepted for publication in the International Journal of Mass Spectrometry ("Author's Accepted Manuscript"). Changes resulting from the publishing process, such as editing, corrections, structural formatting, and other quality control mechanisms, are not reflected in this document. A definitive version was subsequently published in the International Journal of Mass Spectrometry [Volume 363, Pages 8-15 (15 April 2014)]; DOI: 10.1016/j.ijms.2014.02.005.

\section{Downloaded from}

http://scholarscompass.vcu.edu/chem_pubs/10

This Article is brought to you for free and open access by the Dept. of Chemistry at VCU Scholars Compass. It has been accepted for inclusion in Chemistry Publications by an authorized administrator of VCU Scholars Compass. For more information, please contact libcompass@vcu.edu. 
NOTICE: This is the author's version of a work that was accepted for publication in the International Journal of Mass Spectrometry ("Author's Accepted Manuscript"). Changes resulting from the publishing process, such as editing, corrections, structural formatting, and other quality control mechanisms, are not reflected in this document. A definitive version was subsequently published in the International Journal of Mass Spectrometry [Volume 363, Pages 8-15 (15 April 2014)]; DOI: 10.1016/j.ijms.2014.02.005.

\section{A novel two-laser interface for coupling capillary electrochromatography with ion-trap time-of- flight mass spectrometry}

David C. Simpson*,1, Alexander J. Yates, John H. Knox, and

Pat R.R. Langridge-Smith

University of Edinburgh, School of Chemistry, West Mains Road, Edinburgh, EH9 3JJ, Scotland

1Present address: Virginia Commonwealth University, Department of Chemistry, Richmond, VA 23284, USA

*To whom correspondence should be addressed:

David C. Simpson

Virginia Commonwealth University

Department of Chemistry

P.O. Box 842006

Richmond, VA 23284-2006, USA

Email: dsimpson3@vcu.edu

Tel: $\quad+1-804-617-7432$

Keywords: capillary electrochromatography; two-step laser mass spectrometry; iontrap; time-of-flight; mass spectrometry; polycyclic aromatic hydrocarbon

Abbreviations: CEC, capillary electrochromatography; EOF, electroosmotic flow; ESI, electrospray ionization; ID, inner diameter; L ${ }^{2} \mathrm{MS}$, two-step laser mass spectrometry; LC, liquid chromatography; MS, mass spectrometry; $\mathrm{PAH}$, polycyclic aromatic hydrocarbon; REMPI, resonance-enhanced multi-photon ionization; SCX, strong cation exchange;

TOF, time-of-flight 


\section{Abstract}

An interface has been developed for the hyphenation of capillary electrochromatography (CEC) with mass spectrometry (MS). Chromatographic eluate vaporization and selective analyte ionization occur within a quadrupole ion-trap, which permits significant instrument simplification when compared with the atmospheric pressure interfaces typically used for CEC-MS. Vaporization is achieved using laser desorption at $1064 \mathrm{~nm}$ while ionization is accomplished through UV photoionization. This two-step approach, through ionization laser wavelength selection, can provide ultratrace analysis with high selectivity. The mass spectrometer is a hybrid ion-trap time-of-flight (TOF) instrument in which the ion-trap is used in radio frequency-only mode, with DC-pulse ejection, to provide decoupling of the different timescales required for CEC separation and TOF mass analysis. The ion-trap is capable of accumulating ions over multiple laser shots. The mass resolution of the demonstration instrument was circa 1500. Preliminary CECMS runs have been recorded for mixtures containing polycyclic aromatic hydrocarbons. A concentration detection limit of $500 \mathrm{nM}$, for naphthalene in acetonitrile, has been determined for the interface. 


\section{Introduction}

Capillary electrochromatography (CEC) can be described as electroosmotic flow (EOF)driven liquid chromatography (LC). EOF-drive has two significant advantages over pressure-drive. First, the plug flow profile found in EOF-driven LC results in reduced band dispersion when compared with the parabolic flow profile found in pressure-driven LC. Second, band dispersion-minimizing reductions in stationary phase support particle size, which would not place any additional demands on EOF-drive instrumentation, require increased applied pressures in pressure-drive LC [1]. However, these advantages have not yet resulted in widespread popularity [2,3], despite the reporting of numerous CEC applications [4-7]. A key CEC disadvantage is that mobile phase composition influences flow rate, thus adding an extra layer of complexity to method development. Furthermore, when interfacing to mass spectrometry (MS), finding a mobile phase that is ideal for both separation and interface is particularly challenging.

Gordon et al. were first to report the interfacing of CEC to MS, using a continuous flowfast atom bombardment interface [8]. The earlier report of Verheij et al. is best regarded as describing a pressure-drive system in which an applied potential was used to add an electrophoretic component to the separation [9]. In contrast to the earliest work, most reported CEC-MS interfaces incorporate electrospray ionization (ESI). However, while ESI-based interfaces where eluate is sprayed directly into the mass spectrometer have been reported [10], the incompatibility of typical CEC mobile phases with ESI necessitates the addition of an intermediate step where column eluate is diluted in a compatible make-up flow. The most common means of adding make-up flow is the 
sheath flow method [11]. Practical operation of such interfaces for CEC-MS has been described by Lane et al. $[12,13]$. However, since dilution reduces sensitivity and even careful mixing leads to at least some band dispersion, alternative interfacing strategies are clearly required.

Laser resonance-enhanced multi-photon ionization (REMPI) exploits resonant absorption to selectively ionize gas-phase molecules that absorb significantly at a chosen wavelength. Target analytes can be ionized in the presence of high concentrations of non-absorbing background species [14]. Naturally, REMPI must be paired with a method for producing gas-phase neutrals to complete an interface with a liquid-phase separation. The recently introduced atmospheric pressure laser ionization interface combines a heated nebulizer with REMPI at atmospheric pressure [15]; this interface, with the addition of ESI, has also been demonstrated for CEC-MS [16]. However, REMPI is most often used under vacuum with laser desorption, which can be used to provide plumes of vaporized neutrals. Long-wavelength laser desorption produces few ions directly, allowing independent optimization of desorption and ionization processes. This combination is known as two-step laser mass spectrometry (L²MS) [17-19]. While it is possible for the same laser to be used for desorption and ionization, this can lead to complicated mass spectra as reactions occur in the resulting plume of desorbed neutrals and ions [20].

Laser-based mass spectrometry interfaces that operate under vacuum typically operate with solid samples. A notable example of an interface where laser irradiation is applied to a liquid is the laser induced liquid beam ionization/desorption approach [21]. Furthermore, matrix-assisted laser desorption ionization from the hyperbolic inner 
surface of an ion-trap ring electrode has been demonstrated using a solid sample presented on a probe [22] and a flowing liquid probe [23]. Using this concept we have constructed an $\mathrm{L}^{2} \mathrm{MS}$ CEC-MS interface that requires no make-up flow and that avoids the ion transfer losses associated with atmospheric pressure interfaces. The interface is selective for analytes with significant absorbance at the ionization laser wavelength. Desorption and ionization occurs within the quadrupole ion-trap, using cross-bored apertures in the ring electrode to allow laser access. One such aperture is used for presentation of chromatographic eluate. Mass spectra are obtained by ejecting product photoions from the trap into a time-of-flight (TOF) mass analyzer, a configuration chosen chiefly for speed (ejection into the TOF mass analyzer is faster than the scanning out process used in ion-trap mass analysis). Thus, no compromise is required between resolution and scanning speed, and flexibility is retained for options such as switching to higher repetition rate laser systems.

Instrument performance was evaluated with polycyclic aromatic hydrocarbons (PAHs) since they are important environmental pollutants and are amenable to laser ionization at $266 \mathrm{~nm}$. Complex mixtures of PAHs have often been targeted using L ${ }^{2} \mathrm{MS}$ [24]. Chromatographic efficiency was largely maintained, even though a transfer capillary in which pressure-drive conditions existed was used to connect the CEC column terminus to the interface. The use of a transfer capillary was beneficial because it allowed columns to be exchanged without opening the high-vacuum chamber. Expressed as a number of theoretical plates per meter, obtained from measurements of half-height peak widths and retention times, an average chromatographic efficiency of 94,000 was obtained with a test mixture that consisted of acenaphthene, biphenyl, fluorene, 
naphthalene and phenanthrene. A concentration detection limit of $500 \mathrm{nM}$ was obtained for naphthalene in acetonitrile. 


\section{Experimental Procedures}

\section{Materials}

Acenaphthene, biphenyl, naphthalene, and phenanthrene were obtained from SigmaAldrich (Saint Louis, MO, USA); fluorene and ammonium acetate were supplied by BDH (Poole, UK). Acetone, acetonitrile, acetic acid, and methanol were purchased from Fisher Scientific (Pittsburgh, PA, USA); water was double distilled in house. Fused-silica capillaries were from Polymicro Technologies (Phoenix, AZ, USA). Strong cation exchange (SCX) combined with reversed-phase (mixed mode) chromatographic stationary phase ( $3 \mu \mathrm{m}$ particles) was supplied by Hypersil (Runcorn, UK).

\section{Capillary Electrochromatography}

Capillaries were packed largely as described by Boughtflower et al. [25,26]. SCX combined with reversed-phase stationary phase was chosen over reversed-phase alone because the SCX character provides surface charges that increase EOF [27]. Briefly, a slurry of stationary phase particles suspended in acetone was driven rapidly into an empty fused-silica capillary using an applied pressure of 800 bar; a WellChrom K-1900 pneumatic LC pump (Knauer, Berlin, Germany) was used to quickly reach this operating pressure. Stationary phase particle suspension in the packing bomb was maintained using a Miniprobe 55T ultrasonic probe (Kerry Ultrasonics, Hitchin, UK). Particles were initially retained using a Valco (Houston, TX, USA) steel screen (thinner than a frit so reduced resistance to solvent flow). Packing was allowed to proceed for $15 \mathrm{~min}$. After careful depressurization to avoid disturbing the newly packed bed, the system was 
reconfigured without the packing bomb. Water was then passed through the new CEC column for $12 \mathrm{~h}$, using an applied pressure of 800 bar, in an attempt to collapse any voids that formed during the packing process. An electric heating coil (Innovatech, Stevenage, UK) was then used to create a sintered frit at the high-pressure end of the column. The column was reversed for production of the second retaining frit. With this approach a number of short columns can be made simultaneously. The electric heating coil can also be used to create windows for absorbance detection.

A Waters (Milford, MA, USA) Capillary Ion Analyzer was used for stand-alone CEC measurements (214 $\mathrm{nm}$ absorbance detection module installed) and as a high-voltage power supply ( -30 to $+30 \mathrm{kV})$ for CEC-MS. The instrument was equipped with an autosampler. Electrokinetic sample injection was performed by applying a potential of $5 \mathrm{kV}$ for $5 \mathrm{~s}$ while the column inlet was immersed in a vial containing the sample solution. The system required circa $3 \mathrm{~s}$ to ramp up to $5 \mathrm{kV}$, indicating that the injection might be better described as the application of $3.5 \mathrm{kV}$ for $5 \mathrm{~s}$. An Agilent (Palo Alto, CA, USA) 8453 spectrophotometer was used to obtain molar absorptivity constants in mobile phase at $214 \mathrm{~nm}$. To simplify CEC-MS operation, a transfer capillary was used to carry column eluate to the MS-interface. For electrical isolation, a grounded junction was constructed using a Supelco (Bellefonte, PA, USA) Capillary Column Butt Connector, effectively a double-tapered ferrule in a compression unit; electrical connection was achieved by externally coating the inlet end of the transfer capillary with copper using an Edwards (Crawley, UK) E12E4 vacuum coater. Since EOF terminates at the grounded junction, the flow profile in the transfer capillary is parabolic and chromatographic efficiency will be reduced. To minimize this degradation in performance, the transfer 
capillary was much narrower than the separation column [28]. In this work, a CEC column having an inner diameter (ID) of $100 \mu \mathrm{m}$ was paired with a transfer capillary having an ID of $25 \mu \mathrm{m}$.

\section{Mass Spectrometry}

The mass spectrometer and associated power supplies were manufactured by R.M. Jordan (Grass Valley, CA, USA). Michael et al. have described the design and operation of this instrument [29]. For easy access, the source region of the mass spectrometer was housed in a cubic vacuum chamber $(30 \times 30 \times 30 \mathrm{~cm})$ equipped with three fused-silica windows for laser access (windows may be exchanged to allow lasers operating at $\lambda>\sim 3 \mu \mathrm{m}$ to enter the chamber). The source chamber was pumped with a Pfeiffer (Asslar, Germany) TPH2000 2,000 L s${ }^{-1}$ turbomolecular pump, backed by an Edwards E2M40 two-stage rotary pump combined with an Edwards EH250 mechanical booster. This pumping system was chosen to ensure vacuum performance even when pumping relatively large volumes of solvent vapor. The source chamber is separated from the TOF chamber using a gate valve, allowing isolation of the ion-trap from the TOF analyzer. The flight tube of the TOF analyzer is equipped with a liner that allows it to be held at a specified electrical potential relative to ground. By holding the liner at a negative high potential, the positive high potentials that must be applied to other electrodes are reduced, minimizing the risk of arcing to electrically grounded parts of the instrument. The incorporation of a gate valve isolating the ion-trap from the TOF analyzer required some modification to the extraction optics and flight tube liner. The TOF analyzer is pumped with an Edwards EXT250 $250 \mathrm{~L} \mathrm{~s}^{-1}$ turbomolecular pump backed by an Edwards E2M18 two stage rotary pump. The ultimate vacuum (no solvent 
input) obtained in the source chamber was $5 \times 10^{-8} \mathrm{mbar}$, while that obtained in the TOF chamber was $2 \times 10^{-8} \mathrm{mbar}$. Typical working pressures (solvent flow at typical CEC volumetric flow rates) were $2 \times 10^{-6}$ and $4 \times 10^{-7} \mathrm{mbar}$, respectively. These pressures were measured using Penning gauges.

Center axes for the cross-bored, cylindrical apertures in the ion-trap ring electrode were located in the equatorial plane. The two axes make a perpendicular intersection at the center of the trap while the ring electrode was mounted such that one cross-aperture was horizontal and the other was vertical. The diameter of the bored apertures was 0.094 inches $(2.4 \mathrm{~mm})$ and the ID of the ring electrode at the equatorial plane was 0.785 inches $(19.9 \mathrm{~mm})$. A transfer capillary carrying eluate from the CEC column was terminated in the lower aperture of the vertical pair, the terminus flush with the ring electrode surface; a ceramic holder was used for centering and to ensure electrical isolation. An IR desorption laser was fired down the vertical axis to strike the transfer capillary terminus while a UV ionization laser was fired along the horizontal axis as illustrated schematically in Figure 1. The ion-trap was used in radio frequency-only mode as an ion storage and accumulation device; the amplitude of the trapping potential used was $1525 \mathrm{~V}$ (peak-peak). Tandem mass spectrometry was not employed. Collisional cooling of the product photoions results in a cloud of ions having a narrow range of kinetic energies, which is a requirement for effective TOF-MS. During ion ejection, the trapping potential was switched off and an extraction pulse of $-193 \mathrm{~V}$ was applied to the ion-trap end cap electrode closest to the TOF mass analyzer. 


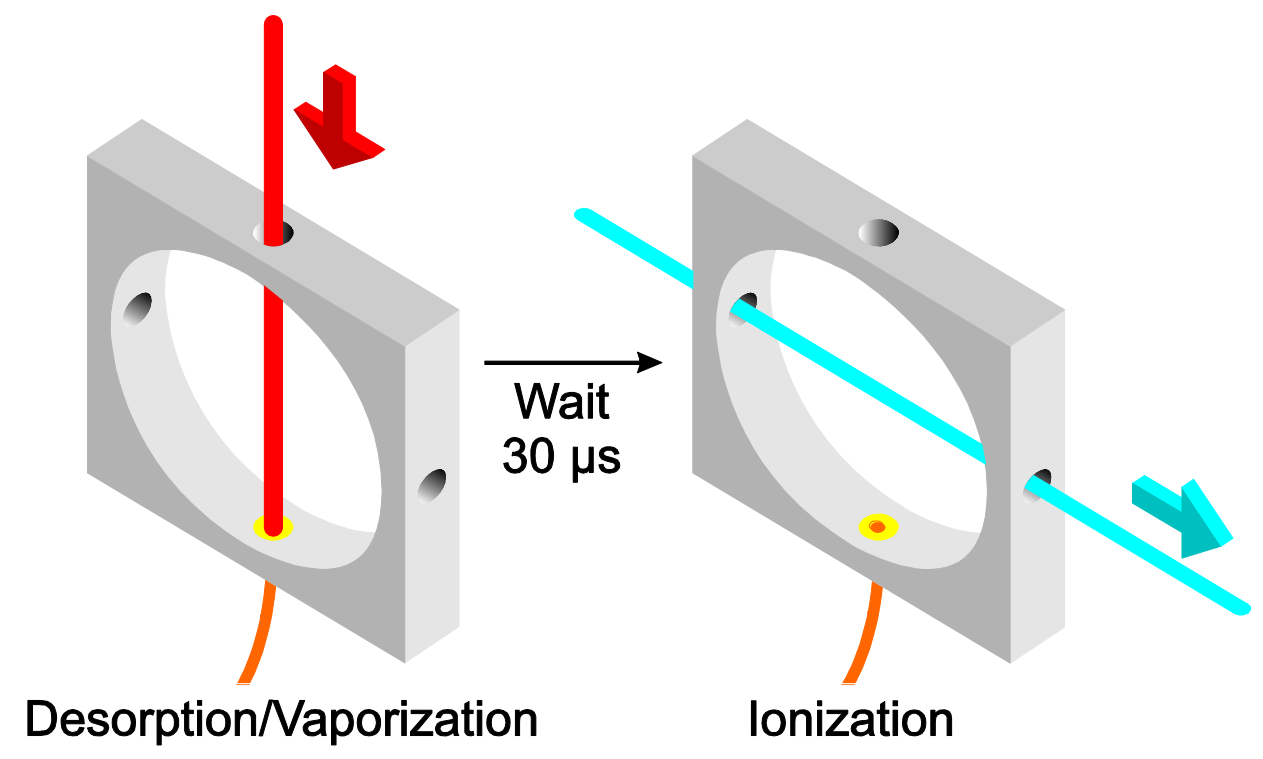

Figure 1: Schematic of the laser desorption laser ionization CEC-MS interface showing how the lasers are used within the ion-trap (IR laser beam for desorption; UV laser beam for ionization); the ring electrode shape has been simplified and the sample position is indicated by the capillary entering from below. 
Laser powers were monitored using a Molectron (Portland, OR, USA) PM500A power meter in conjunction with a Molectron PM10V1 probe. Desorption was achieved using a Continuum (Santa Clara, CA, USA) Minilite Nd:YAG laser operating at $1064 \mathrm{~nm}$; the laser was aligned using a quartz prism, followed by a lens and an iris before entering the vacuum chamber through a fused-silica window. The repetition rate was fixed at $10 \mathrm{~Hz}$ and laser power was tuned to maximize desorption while not providing sufficient power to effect non-resonant multi-photon ionization. With the meter placed below the trap and with the transfer capillary and holder removed, laser power was measured at circa $60 \mathrm{~mW}$. Ionization was achieved using a Quantel (Les Ulis, France) Brilliant Nd:YAG laser equipped with harmonic generation modules to provide $266 \mathrm{~nm}$ laser pulses at a repetition rate of $10 \mathrm{~Hz}$. Two quartz prisms were used to align the beam through a lens and a fused-silica window into the vacuum chamber. Laser power measured at the beam dump (a fused-silica window allowed the beam to exit the vacuum chamber after passing through the trap) was measured at circa $40 \mathrm{~mW}$.

Experimental timing was controlled using an EG\&G (Wokingham, UK) 9650A fourchannel delay generator. The lasers fixed the experimental repetition rate at $10 \mathrm{~Hz}$. Laser ionization occurred $30 \mu \mathrm{s}$ after laser desorption to allow the plume of desorbed neutrals to expand into the space irradiated by the ionization laser. The duration of this delay was determined by tuning for maximum signal intensity. A second delay generator, a Stanford Research Systems (Sunnyvale, CA, USA) DG535, was added for ion accumulation over more than one laser cycle. For instrument tuning and characterization, a leak inlet was employed that consisted of a long length of fused-silica capillary terminated at the ion-trap ring electrode in the same way as the transfer 
capillary; the length $(564 \mathrm{~mm})$ and ID $(25 \mu \mathrm{m})$ of the leak inlet capillary were chosen to provide similar flow rates to the CEC system. Volumetric flow rates for the leak inlet were estimated using the Hagen-Poiseuille equation. Mass spectra were recorded using a LeCroy (Chestnut Ridge, NY, USA) 9350M 500 MHz digital oscilloscope. For collection of chromatographic data, the oscilloscope was controlled from a computer running LabVIEW (National Instruments, Austin, TX, USA) software. The time required for the transfer of a single mass spectrum (50,000 time points at 8-bit intensity resolution) from the oscilloscope to the computer was approximately $1.3 \mathrm{~s}$. Due to the magnitude of this delay, during chromatographic runs, ten mass spectra were averaged before transfer to the computer, resulting in circa 27 mass spectra being recorded per minute.

In a separate experiment, solvent vaporization at the capillary terminus was observed using a video camera equipped with a macro lens. For viewing, the capillary terminus was moved just inside one of the fused-silica windows ordinarily used to allow lasers to enter the vacuum chamber. Desorption laser optics were adjusted to align the beam to the new target and vacuum chamber pressure was increased to circa $10^{-3}$ mbar to better represent conditions found inside the enclosed ion-trap. Targeting an object placed just inside a vacuum chamber window would be expected to result in scattered laser light exiting the vacuum chamber. Therefore, the area around the window and video camera was carefully enclosed. 


\section{Results}

\section{CEC Test Separation}

Characterization of the interface required a test separation. Operating at $266 \mathrm{~nm}$, the ionization laser was suitable for REMPI of PAHs. Naphthalene, biphenyl, fluorene, acenaphthene and phenanthrene were chosen as test mixture components. CEC separation was optimized without the mass spectrometer, using absorbance detection at $214 \mathrm{~nm}$. Figure 2 shows an example chromatogram. Baseline separation was achieved for all five peaks, with the close spacing of fluorene and acenaphthene providing a quick indication of resolution. Peak identities were determined by sample spiking.

Chromatographic efficiencies for each peak, expressed as plate numbers obtained from measurements of half-height peak width and retention time, are listed in Table 1. The baseline disturbance at circa 6.5 min represents the solvent front, giving the linear mobile phase velocity as $1.1 \mathrm{~mm} \mathrm{~s}^{-1}$. Assuming that the flowing volume of the column is $50 \%$ of the open tube volume, the volumetric flow rate is estimated at $260 \mathrm{~nL} \mathrm{~min}{ }^{-1}$. Using this flow rate along with the peak area for naphthalene $(0.00207 \mathrm{~min})$, the molar absorption coefficient for naphthalene at $214 \mathrm{~nm}$ in mobile phase $\left(57,800 \mathrm{M}^{-1} \mathrm{~cm}^{-1}\right)$, and the detector path length $(100 \mu \mathrm{m})$, the amount of naphthalene injected can be determined to be approximately 1 picomole. Naphthalene was present in the injected solution at $500 \mu \mathrm{M}$, so the injection volume is $2 \mathrm{~nL}$. Alternatively, the injection volume can be obtained by multiplying the volumetric flow rate by the injection time and the injection potential as a proportion of the running potential. Using the averaged value of $3.5 \mathrm{kV}$ over $5 \mathrm{~s}$, a similar result for the injection volume, $2.5 \mathrm{~nL}$, is obtained. 


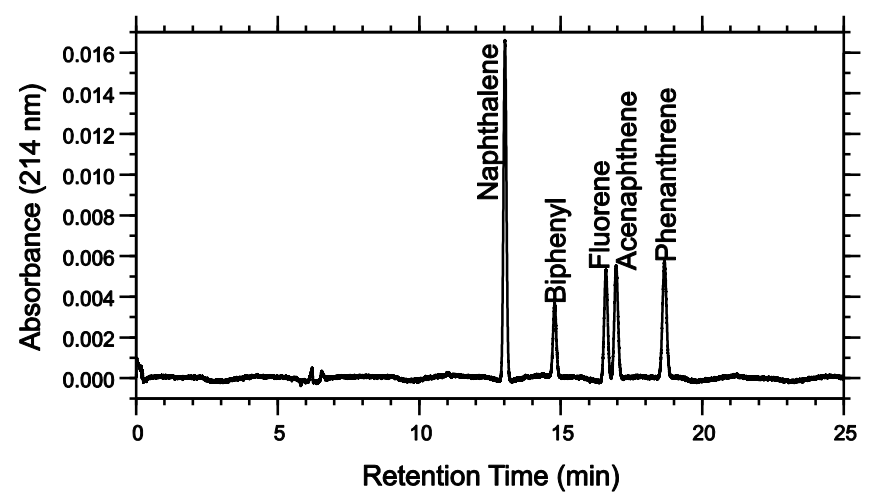

Figure 2: Chromatogram showing the CEC separation of a test mixture containing PAHs. Injection was electrokinetic, achieved by applying a potential of $5 \mathrm{kV}$ for $5 \mathrm{~s}$ while the column inlet was immersed in a vial containing each sample component at $500 \mu \mathrm{M}$ in acetonitrile. The running potential was $30 \mathrm{kV}$ and the mobile phase was $75 \%$ acetonitrile, $25 \% 50 \mathrm{mM}$ aqueous ammonium acetate adjusted to $\mathrm{pH} 6.0$ with acetic acid. Stationary phase was SCX combined with reversedphase (mixed mode) material. Column ID was $100 \mu \mathrm{m}$ and length from inlet to detection window was $432 \mathrm{~mm}$; total length was $624 \mathrm{~mm}$. Chromatographic efficiencies of at least 70,000 theoretical plates were achieved for all five peaks. 
Table 1: Comparison of chromatographic efficiencies obtained using on-column absorbance detection (Figure 2) with those obtained using the hyphenated CEC-MS system (Figure 5a). Chromatographic efficiencies are expressed as plate numbers obtained from measurements of half-height peak widths and retention times. Plate counts per meter are provided to aid comparison between columns of different lengths.

\begin{tabular}{lcc}
\hline $\begin{array}{l}\text { Analyte (listed in } \\
\text { elution order) }\end{array}$ & $\begin{array}{c}\text { Chromatographic Efficiency } \\
\text { for CEC with Absorbance } \\
\text { Detection at 214 nm } \\
\text { (thousands of plates/plates } \\
\text { per meter) }\end{array}$ & $\begin{array}{c}\text { Chromatographic } \\
\text { Efficiency for the } \\
\text { Hyphenated CEC-MS } \\
\text { System (thousands of } \\
\text { plates/plates per meter) }\end{array}$ \\
\hline Naphthalene & $70 / 162$ & $55 / 100$ \\
Biphenyl & $73 / 168$ & $66 / 121$ \\
Fluorene & $76 / 176$ & $32 / 58$ \\
Acenaphthene & $73 / 170$ & $80 / 147$ \\
Phenanthrene & $71 / 162$ & $24 / 44$ \\
\hline
\end{tabular}




\section{Behavior of Liquids and Solutions Entering the lon-Trap}

Desorption in $\mathrm{L}^{2} \mathrm{MS}$ is most commonly performed using $10.6 \mu \mathrm{m}$ laser irradiation. However, using this wavelength resulted in damage to the fused-silica capillary terminus. Therefore, desorption was carried out at $1064 \mathrm{~nm}$, a wavelength at which fused-silica is transparent and at which no damage to the capillary terminus was observed. However, non-resonant multi-photon ionization becomes more likely at shorter wavelengths, resulting in the imposition of laser power limits when only desorption is required. Such power limits can readily be determined by ramping intensity until ions are detected without the ionization laser. Since the aim of this work was to devise a selective MSinterface, the use of REMPI was essential. Therefore, the ability of the desorption laser to vaporize solvent at lower, $\mathrm{L}^{2} \mathrm{MS}$-compatible, powers $(60 \mathrm{~mW})$ was investigated. Furthermore, a major concern with the introduction of liquids through a narrow capillary into a high-vacuum chamber is that rapid evaporation may lead to flow stoppage through freezing. A flowing MALDI probe has been reported that required an infrared heater to avoid solvent freezing [23]. Therefore, solvent outflow from the capillary terminus was observed under vacuum using a video camera. Without laser irradiation, solvent accumulation was observed (a droplet formed at the tip and excess solvent ran down the outside of the capillary). This behavior was seen in the case of acetonitrile, water and methanol, as well as a solution of naphthalene at $5 \mathrm{mM}$ in acetonitrile. When the $1064 \mathrm{~nm}$ laser was focused onto the end of the capillary at typical experimental power output settings, no solvent accumulation was observed. Figure 3 shows the case where an acetonitrile droplet was allowed to form by using a shutter to block the laser path, followed by the shutter being opened to permit the laser to strike capillary terminus. 

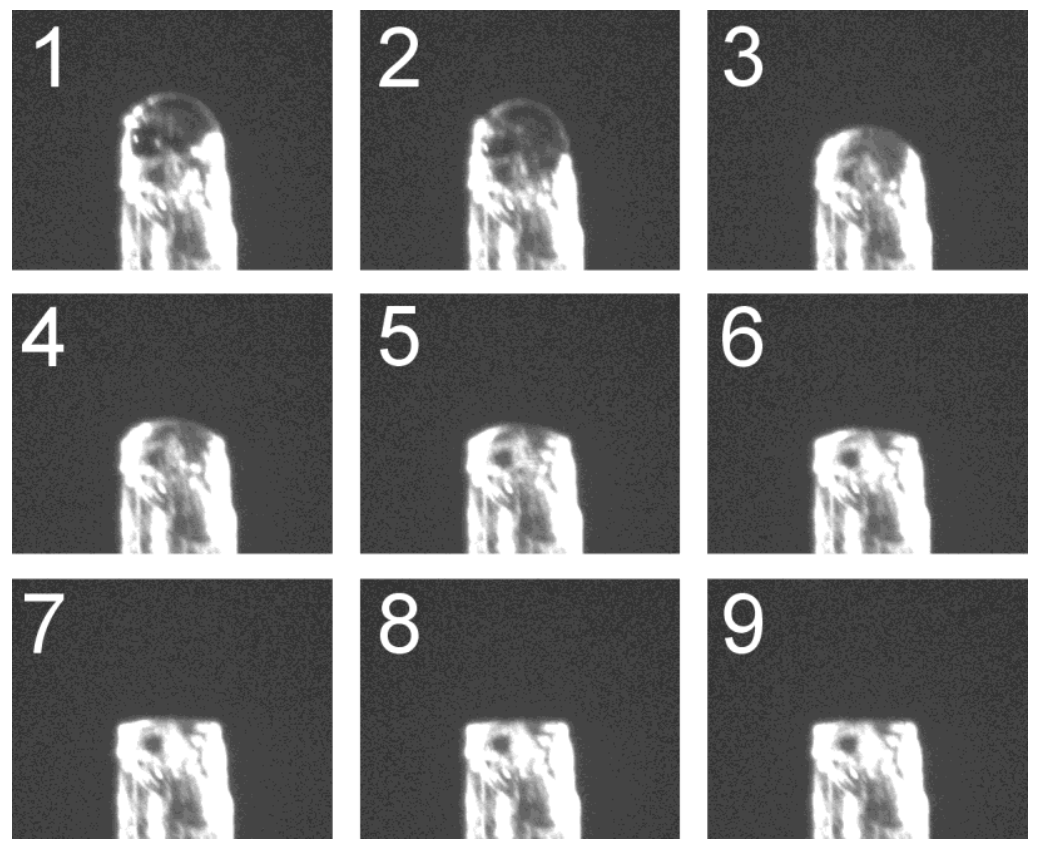

Figure 3: Filmstrip, each frame separated by $0.2 \mathrm{~s}$, showing the effect of laser irradiation (1064 $\mathrm{nm}$ ) on an acetonitrile droplet at an ambient pressure of circa $10^{-3} \mathrm{mbar}$ (typical ion-trap operating pressure). The laser path was blocked to allow the droplet shown in Frame 1 to form. For scale, the width of the capillary is circa $400 \mu \mathrm{m}$. 


\section{Interface Characterization using the Leak Inlet}

Characterization of the MS-interface using eluting peaks from CEC separations was difficult due to the transient nature of the signal, resulting in the adoption of a leak interface capable of providing a constant stream of analyte. Naphthalene in acetonitrile at various concentrations was used as test solution, flowing to the ion-trap at a

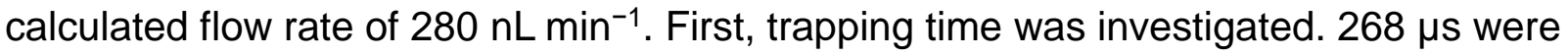
required from initializing the cycle through to the ionization laser pulse and a further $100 \mu \mathrm{s}$ were required for recording a TOF mass spectrum, so trapping times close to the full laser cycle time of $100 \mathrm{~ms}$ were available. Figure 4a illustrates the variation in signal intensity (expressed as peak area for a fixed integration window centered on the molecular ion) observed for naphthalene at $500 \mu \mathrm{M}$ in acetonitrile when using trapping times ranging from $10 \mu \mathrm{s}$ to $90 \mathrm{~ms}$. Similarly, Figure $4 \mathrm{~b}$ shows the trend for $5 \mathrm{mM}$ naphthalene in acetonitrile. At both concentrations, using very short trapping times results in poor signals. Longer trapping times result in increased signal until a plateau is reached, with the plateau achieved at a shorter trapping time for the higher concentration. However, a drop in molecular ion signal for the longest trapping times is seen for the higher concentration. 

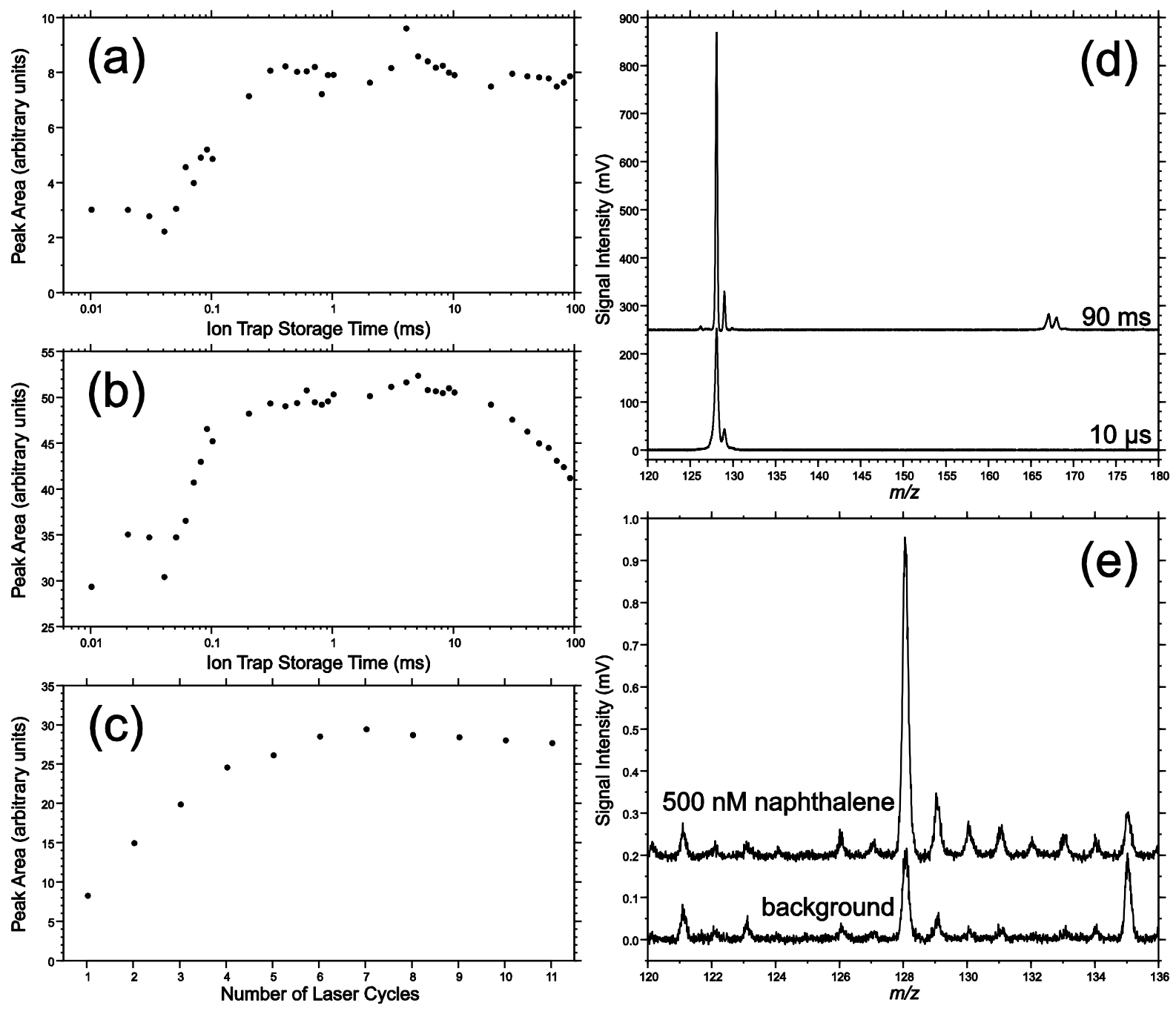

Figure 4: Interface performance characteristics obtained using the leak inlet operating at a flow rate of $280 \mathrm{~nL} \mathrm{~min}{ }^{-1}$. Each mass spectrum and data point results from the combination of 100 mass spectra. First, the effect of varying ion-trap storage time on molecular ion peak area is shown for $500 \mu \mathrm{M}$ (a) and $5 \mathrm{mM}$ (b) naphthalene in acetonitrile. Second, the impact on molecular ion peak area of storing ions over a number of laser cycles, with the delay between the final laser pulse and ion ejection fixed at $10 \mathrm{~ms}$, is displayed for $500 \mu \mathrm{M}$ naphthalene in acetonitrile (c). Mass spectra for $10 \mu \mathrm{s}$ and $90 \mathrm{~ms}$ ion-trapping times for $5 \mathrm{mM}$ naphthalene in acetonitrile, with the $\mathbf{9 0} \mathrm{ms}$ trapping time data displaced $+250 \mathrm{mV}$ for clarity, are also provided (d). Finally, a mass spectrum obtained for $500 \mathrm{nM}$ naphthalene in acetonitrile, with the data displaced $+0.2 \mathrm{mV}$ for clarity, is compared with that for acetonitrile alone (e). 
Signal increase over that found at the shortest trapping times is due to collisional cooling. lons will be produced along the laser path from side to side of the trapping space, resulting in some time being required to concentrate ions toward the center of the trap for efficient extraction to the mass analyzer. Initially, helium was added, as is normal in ion-trap instruments, but on tuning it was found that helium was unnecessary. Solvent vapor alone appeared to provide sufficient cooling. However, Figure 4d illustrates the disadvantage of using solvent vapor for ion cooling, indicating why the naphthalene molecular ion signal decreases at the longest trapping times in Figure $4 \mathrm{~b}$. It can be seen that at 90 ms trapping time a new peak has formed at $m / z=167$, consistent with the formation of an acetonitrile adduct. In contrast, the $10 \mu$ s trapping time spectrum shows no adduct, but the naphthalene peak is much wider and smaller due to insufficient time having been allowed for cooling and concentration of the ion cloud. Since splitting analyte signal between two peaks is not beneficial, but reducing peak widths and increasing intensities is advantageous, a fixed trapping time of $10 \mathrm{~ms}$ was selected for CEC-MS experiments.

The leak inlet analyte introduction system was also used to estimate a concentration detection limit for the system. As illustrated in Figure 4e, this was found to be in the region of $500 \mathrm{nM}$ for naphthalene in acetonitrile. Sample consumption at this concentration was 0.23 femtomoles per laser shot (100 laser shots were averaged to produce Figure 4e). Accumulation of ions over a number of laser cycles is an obvious way to improve interface sensitivity. Using $500 \mu \mathrm{M}$ naphthalene in acetonitrile entering through the leak inlet at $280 \mathrm{~nL} \mathrm{~min}{ }^{-1}$, the effect of increasing cycle count on molecular ion peak area was observed. Storage time between the final laser pulse and ion ejection 
was fixed at $10 \mathrm{~ms}$. In Figure 4c, peak area can be seen to rise steadily up to circa seven cycles and then stay approximately constant for higher numbers of laser cycles. Maximum peak area of approximately 30 arbitrary units at seven cycles (versus 8 arbitrary units for one cycle) does not simply indicate that the trap was full; larger peak areas were found when $5 \mathrm{mM}$ naphthalene in acetonitrile was used in the leak inlet as shown in Figure 4b (peak area units are arbitrary because they are calculated from micro-channel plate detector output voltages and flight times, but they can be compared). However, although at first sight this gain in sensitivity appears useful, ions created by earlier shots and concentrated into the center of the trap will be repeatedly irradiated by both laser beams. Such heating of these ions can result in dissociation, complicating the mass spectra produced. Since retaining simple mass spectra dominated by molecular ions was an advantage for CEC-MS, multiple accumulation cycles were not used.

\section{Hyphenated CEC-MS System Performance}

Figure 5a shows CEC-MS selected ion chromatograms for the separation of a test mixture consisting of naphthalene, biphenyl, fluorene, acenaphthene and phenanthrene (biphenyl and acenaphthene are isobaric and hence share the same chromatogram), each present at $5 \mathrm{mM}$. A comparison of chromatographic efficiencies obtained from the data illustrated in Figure 5a with those achieved using on-column absorbance detection (illustrated in Figure 2) is presented in Table 1. All plate counts were calculated from measurements of half-height peak widths and retention times. While chromatographic efficiencies were fairly constant for on-column detection, those for MS vary considerably and were on average about $55 \%$ of those for absorbance detection. Efficiencies are 
especially low for fluorene and phenanthrene due to increased tailing when compared with the other test mixture components. Similar CEC-MS peak asymmetry and resolution were seen when each test mixture component was present at $500 \mu \mathrm{M}$, as illustrated in Figure 5b. Retention times are somewhat shorter when compared with the separation in Figure 2 because the running potential of $30 \mathrm{kV}$ was applied from the column inlet to the grounded junction (546 $\mathrm{mm}$ ) in the CEC-MS case versus across both the packed bed and the open section of tubing beyond the detection window (combined length $624 \mathrm{~mm}$ ) in the absorbance detection case.

Since the aim of this work was to create a selective analytical system for the detection of targeted compounds from complex matrices, conditions were sought that resulted mostly in the production of molecular ions and hence simple mass spectra. However, while PAH radical cations are resilient toward decomposition, fragmentation through acetylene and hydrogen atom neutral losses does occur [30]. Figure $5 c$ shows the mass spectrum obtained at the selected ion chromatogram peak maximum for phenanthrene from the same CEC-MS run used to construct Figure 5a. Fragmentation through the neutral loss of acetylene, to produce the biphenylene radical cation [31], is prominent. Acetylene loss was also seen for naphthalene, while hydrogen atom neutral losses were observed for fluorene and acenaphthene. Since fragmentation could result in misleading mass spectra for co-eluting species, the benefits of efficient chromatography are clear. 

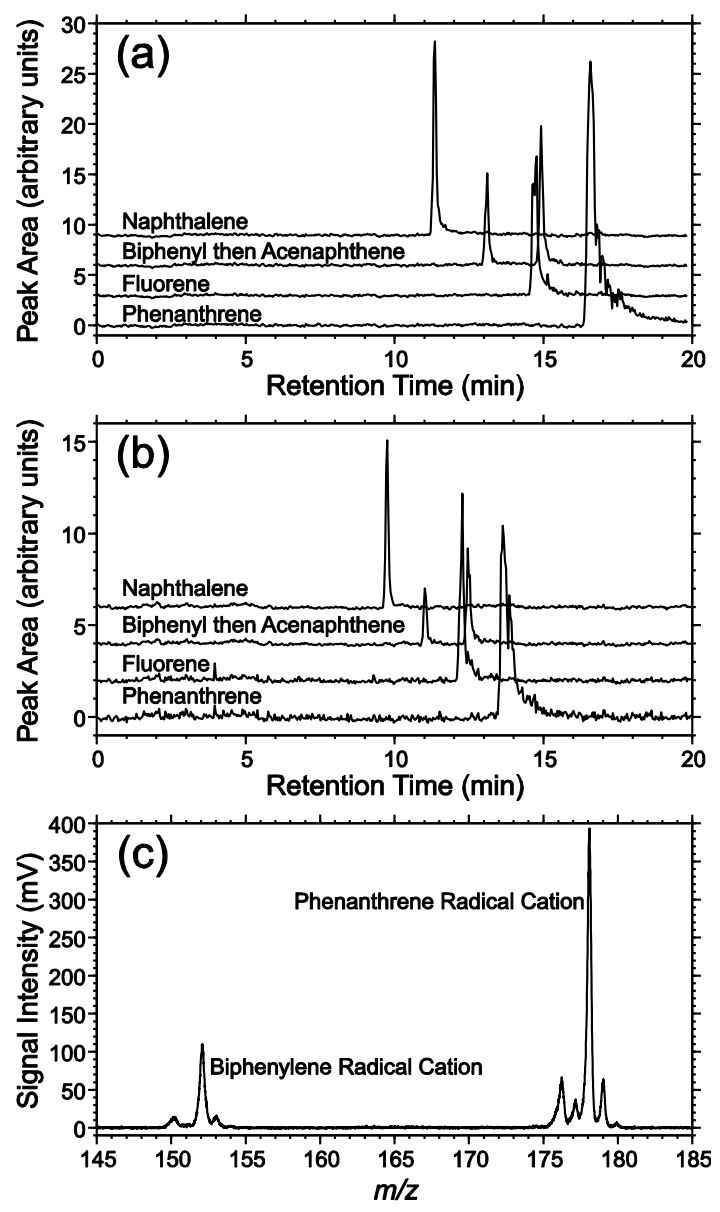

Figure 5: Selected ion chromatograms, displaced vertically in $\mathbf{3}$ (a) or $\mathbf{2}$ (b) arbitrary unit steps for clarity and with isobaric biphenyl and acenaphthene sharing the same chromatogram, for the CECMS separation of a test mixture containing each sample component at $5 \mathrm{mM}$ (a) or $500 \mu \mathrm{M}$ (b) in acetonitrile. Electrokinetic injection was achieved by applying a potential of $5 \mathrm{kV}$ for $5 \mathrm{~s}$ while the column inlet was immersed in the sample vial. The running potential was $30 \mathrm{kV}$ and the mobile phase was $75 \%$ acetonitrile, $25 \% 50 \mathrm{mM}$ aqueous ammonium acetate adjusted to $\mathrm{pH} 6.0$ with acetic acid. Stationary phase was SCX combined with reversed-phase (mixed mode) material. Column dimensions were $100 \mu \mathrm{m}$ ID $\times 546 \mathrm{~mm}$, while transfer capillary dimensions were $25 \mu \mathrm{m}$ ID $\times$ $540 \mathrm{~mm}$. Chromatographic resolution is lower than that found for absorbance detection, but fluorene and acenaphthene are still distinct. Acetylene and hydrogen atom neutral losses were observed for some test mixture components, with the fragmentation of phenanthrene to produce biphenylene being particularly prominent (c). 


\section{Discussion}

A new CEC-MS interface based on L'S has been demonstrated using the separation of a small number of PAHs. Due to the isocratic chromatography and small column volumes employed, maximum injection volumes compatible with high chromatographic performance were in the low $\mathrm{nL}$ range. Naturally, such small injection volumes limit sensitivity (except for cases, such as single cell analyses, where only small volumes are available). The much larger injection volumes (often in the low- $\mu \mathrm{L}$ range) typically encountered in pressure-drive LC, using similarly sized columns, are made possible by gradient elution. While gradient elution-CEC was first reported in the mid-1990s [32,33], intricate equipment and the challenges of working with a system where EOF rates change with mobile phase composition through the course of a run discourage widespread adoption. While mobile phases designed to generate constant flow rates under gradient conditions have recently been reported [34], further limiting flexibility in method development is not desirable. In general, developing an effective separation in a system where mobile phase composition directly influences flow rate is perhaps the greatest drawback of CEC.

Reduced chromatographic resolution for CEC-MS when compared with on-column absorbance detection CEC could have multiple causes. Additional band dispersion originating from the pressure-drive conditions found between the column terminus and the ion-trap and imperfect assembly of the grounded junction would be expected to impact all test mixture components. Shortening the transfer capillary would be expected to result in improved resolution, but vacuum chamber size and the desire to keep the 
grounded junction accessible, to allow columns to be exchanged without opening the chamber, precluded this simple solution. However, these causes of band dispersion cannot explain the differential peak tailing observed for the heaviest test mixture components, fluorene and phenanthrene. Overloading of fluorene and phenanthrene seems unlikely as similar chromatographic peak shapes are observed at $5 \mathrm{mM}$ (Figure $5 \mathrm{a}$ ) and $500 \mu \mathrm{M}$ (Figure $5 \mathrm{~b}$ ), but adsorption to the bare fused-silica inside walls and terminus of the transfer capillary could be important since the larger molecules, having greater polarizability, would be more likely to interact with the surface. Therefore, tailing for the heavier test mixture components might best be investigated by using coated capillaries to reduce adsorption, although sufficient robustness to survive laser irradiation at the terminus could prove challenging.

Non-chromatographic sources of peak asymmetry must also be considered. Persistence of desorbed neutrals in the ion-trap seems unlikely given the supersonic rate at which the plume of desorbed neutrals expands. Desorbed neutrals not ionized when intended are expected to be dispersed before the next laser shot. However, incomplete laser desorption is possible. Any material not desorbed would remain at the transfer capillary terminus, carrying over to the next laser desorption event. The lighter test mixture components would more readily evaporate, explaining the enhanced tailing observed for heavier fluorene and phenanthrene. Moving to $10.6 \mu \mathrm{m}$ laser desorption could perhaps be beneficial, but a carefully designed assembly would be required to protect the fusedsilica capillary terminus while avoiding the introduction of dead volumes detrimental to separation performance.

While the interface was designed around the compatibility of CEC flow rates with the 
vacuum pumping capacity of the instrument, pressure-drive LC systems that can reliably supply similar flow rates have recently become commonplace. There is no reason why the interface could not be combined with these systems or indeed with any separation method having appropriate flow rate and eluate composition. The key advantage of the interface is the selectivity that arises from ionization laser wavelength choice, which offers the possibility of detecting a target analyte among high concentrations of nonabsorbing species. Furthermore, being able to analyze target compounds directly in complex matrices offers the possibility of simplified sample preparation procedures, thus reducing total analysis time and the chance of incorporating biases into methods. In contrast, for most other interfaces, little can be done to target particular analytes.

The instrumental system also offers the possibility of enhanced sensitivity through achieving an effective $100 \%$ duty cycle. Most separations provide a continuous stream of eluate and are coupled with continuous ion sources, such as ESI, while most MS instruments can only accept ions for a small proportion of the total cycle time. For example, instruments based on orthogonal-acceleration TOF mass analyzers typically have duty cycles in the range from $5 \%$ to $30 \%$ [35]. The L ${ }^{2} \mathrm{MS}$ interface reported here is capable of an effective $100 \%$ duty cycle because eluate simply collects at the capillary terminus between laser shots. No material should be lost, although volatile compounds might be depleted by evaporation. Sensitivity is also enhanced by creating ions inside the trap, thus avoiding transmission losses from a distant source. However, sensitivity will be lost due to the ionization laser beam only irradiating part of the trap volume.

Interface characterization using the chromatographic system was difficult due to the transient nature of eluting peaks, so performance was assessed using a leak inlet that 
was designed to provide a continuous stream of analyte at flow rates mimicking those of the separation. Generally, the interface performed as expected: collisional cooling time was required to focus ions toward the center of the trap and sensitivity could be improved by accumulating ions over a number of laser cycles. What was not originally anticipated was the role of solvent vapor. It was quickly determined that helium was not required for trapping, presumably due to sufficient cooling being achieved with solvent vapor alone. The simplest way to regain control of ion-trap operating pressure would be to lower the flow rate sufficiently so that helium would be required to augment solvent vapor. The amount of helium added could then be tuned for optimum performance. Furthermore, reducing the amount of solvent vapor present would reduce adduction, improving the viability of accumulation over multiple laser cycles. However, maintaining chromatographic performance while reducing flow rate would require a reduction in column ID, which would lead to a concomitant reduction in injection volume and thus sensitivity.

In conclusion, an interface between liquid phase separations and mass spectrometry has been demonstrated that is designed around the concept of selective laser ionization of target compounds in the presence of high concentrations of non-absorbing species. Many performance-enhancing improvements could be envisioned, such as a data system fast enough to remove the need for spectrum averaging and adjustments to the vacuum chamber to allow the use of shorter transfer capillaries. Tailing, which was observed for the heaviest test mixture components and was presumably due to adsorption to fused-silica surfaces or incomplete desorption from the transfer capillary terminus, is more problematic; however, switching to an appropriately coated transfer 
capillary and careful selection of desorption laser wavelength and power for each system is likely to be beneficial. 


\section{Acknowledgements}

We especially thank Robert J. Boughtflower for assistance with various aspects of CEC, David P.A. Kilgour for LabVIEW programming, and Robert R.J. Maier for his help in the design and construction of the mass spectrometer. This work was supported by the Engineering and Physical Sciences Research Council [grant number GR/L32460/01]. D.C.S. received a Collaborative Awards in Science and Engineering studentship sponsored by Imperial Chemical Industries. 


\section{References}

[1] J.H. Knox, I.H. Grant, Miniaturisation in pressure and electroendosmotically driven liquid chromatography: Some theoretical considerations, Chromatographia 24 (1987) 135-143.

[2] F. Svec, R.E. Majors, What ever happened to capillary electrochromatography? LCGC North Am. 27 (2009) 1032-1039.

[3] F. Svec, CEC: Selected developments that caught my eye since the year 2000, Electrophoresis 30 (2009) S68-S82.

[4] G. Vanhoenacker, T. Van den Bosch, G. Rozing, P. Sandra, Recent applications of capillary electrochromatography, Electrophoresis 22 (2001) 4064-4103.

[5] S. Eeltink, G.P. Rozing, W.Th. Kok, Recent applications in capillary electrochromatography, Electrophoresis 24 (2003) 3935-3961.

[6] W.Th. Kok, S. Eeltink, Recent applications in capillary electrochromatography, Electrophoresis 27 (2006) 84-96.

[7] Y. Huo, W.Th. Kok, Recent applications in CEC, Electrophoresis 29 (2008) 80-93.

[8] D.B. Gordon, G.A. Lord, D.S. Jones, Development of packed capillary column electrochromatography/mass spectrometry, Rapid Commun. Mass Spectrom. 8 (1994) 544-548.

[9] E.R. Verheij, U.R. Tjaden, W.M.A. Niessen, J. van der Greef, Pseudoelectrochromatography-mass spectrometry: A new alternative, J. Chromatogr. 554 (1991) 339-349.

[10] G.A. Lord, D.B. Gordon, P. Myers, B.W. King, Tapers and restrictors for capillary electrochromatography and capillary electrochromatography-mass spectrometry, J. Chromatogr. A 768 (1997) 9-16.

[11] R.D. Smith, C.J. Barinaga, H.R. Udseth, Improved electrospray ionization interface for capillary zone electrophoresis-mass spectrometry, Anal. Chem. 60 (1988) 1948-1952.

[12] S.J. Lane, R. Boughtflower, C. Paterson, T. Underwood, Capillary electrochromatography/mass spectrometry: Principles and potential for application in the pharmaceutical industry, Rapid Commun. Mass Spectrom. 9 (1995) 12831287. 
[13] S.J. Lane, R. Boughtflower, C. Paterson, M. Morris, Evaluation of a new capillary electrochromatography/mass spectrometry interface using short columns and high field strengths for rapid and efficient analyses, Rapid Commun. Mass Spectrom. 10 (1996) 733-736.

[14] U. Boesl, Laser mass spectrometry for environmental and industrial chemical trace analysis, J. Mass Spectrom. 35 (2000) 289-304.

[15] M. Constapel, M. Schellenträger, O.J. Schmitz, S. Gäb, K.J. Brockmann, R. Giese, et al., Atmospheric-pressure laser ionization: A novel ionization method for liquid chromatography/mass spectrometry, Rapid Commun. Mass Spectrom. 19 (2005) 326-336.

[16] S. Droste, M. Schellenträger, M. Constapel, S. Gäb, M. Lorenz, K.J. Brockmann, et al., A silica-based monolithic column in capillary HPLC and CEC coupled with ESI-MS or electrospray-atmospheric-pressure laser ionization-MS, Electrophoresis 26 (2005) 4098-4103.

[17] R. Zenobi, In situ analysis of surfaces and mixtures by laser desorption mass spectrometry, Int. J. Mass Spectrom. Ion Process. 145 (1995) 51-77.

[18] M.S. de Vries, Two step laser mass spectrometry, Rev. Anal. Chem. 19 (2000) 269-287.

[19] H.-L. Wang, Y.-J. Hu, D. Xing, Recent progress of two-step laser desorption/laser ionization mass spectrometry and its application, Chin. J. Anal. Chem. 39 (2011) 276-282.

[20] A.E. Pomerantz, M.R. Hammond, A.L. Morrow, R.N. Zare, O.C. Mullins, Two-step laser mass spectrometry of asphaltenes, J. Am. Chem. Soc. 130 (2008) 72167217.

[21] W. Kleinekofort, J. Avdiev, B. Brutschy, A new method of laser desorption mass spectrometry for the study of biological macromolecules, Int. J. Mass Spectrom. lon Process. 152 (1996) 135-142.

[22] B.M. Chien, S.M. Michael, D.M. Lubman, Enhancement of resolution in matrixassisted laser desorption using an ion-trap storage/reflectron time-of-flight mass spectrometer, Rapid Commun. Mass Spectrom. 7 (1993) 837-843.

[23] L. He, L. Liang, D.M. Lubman, Continuous-flow MALDI mass spectrometry using an ion trap/reflectron time-of-flight detector, Anal. Chem. 67 (1995) 4127-4132.

[24] J.E. Elsila, N.P. de Leon, R.N. Zare, Factors affecting quantitative analysis in laser desorption/laser ionization mass spectrometry, Anal. Chem. 76 (2004) 2430-2437. 
[25] R.J. Boughtflower, T. Underwood, C.J. Paterson, Capillary electrochromatography-Some important considerations in the preparation of packed capillaries and the choice of mobile phase buffers, Chromatographia 40 (1995) 329-335.

[26] R.J. Boughtflower, T. Underwood, J. Maddin, The production of packed capillaries using a novel pressurised ultrasound device, Chromatographia 41 (1995) 398402 .

[27] N. Smith, M.B. Evans, Comparison of the electroosmotic flow profiles and selectivity of stationary phases used in capillary electrochromatography, $\mathrm{J}$. Chromatogr. A 832 (1999) 41-54.

[28] R.J. Boughtflower, C.J. Paterson, J.H. Knox, Control of dispersion in capillary electrochromatography coupled to UV and mass spectrometric detection, J. Chromatogr. A 887 (2000) 409-420.

[29] S.M. Michael, D.M. Lubman, M. Chien, An ion trap storage/time-of-flight mass spectrometer, Rev. Sci. Instrum. 63 (1992) 4277-4284.

[30] Y. Gotkis, M. Oleinikova, M. Naor, C. Lifshitz, Time-dependent mass spectra and breakdown graphs. 17. Naphthalene and phenanthrene, J. Phys. Chem. 97 (1993) 12282-12290.

[31] Y. Ling, J.M.L. Martin, C. Lifshitz, Energetics of acetylene loss from C14H10*+ cations : A density functional calculation, J. Phys. Chem. A 101 (1997) 219-226.

[32] C. Yan, R. Dadoo, R.N. Zare, D.J. Rakestraw, D.S. Anex, Gradient elution in capillary electrochromatography, Anal. Chem. 68 (1996) 2726-2730.

[33] C.G. Huber, G. Choudhary, C. Horváth, Capillary electrochromatography with gradient elution, Anal. Chem. 69 (1997) 4429-4436.

[34] M.W.L. Watson, J.M. Mudrik, A.R. Wheeler, Gradient elution in microchannel electrochromatography, Anal. Chem. 81 (2009) 3851-3857.

[35] A. V Loboda, I. V Chernushevich, A novel ion trap that enables high duty cycle and wide $\mathrm{m} / \mathrm{z}$ range on an orthogonal injection TOF mass spectrometer, J. Am. Soc. Mass Spectrom. 20 (2009) 1342-1348. 


\section{Table of Contents Graphic}

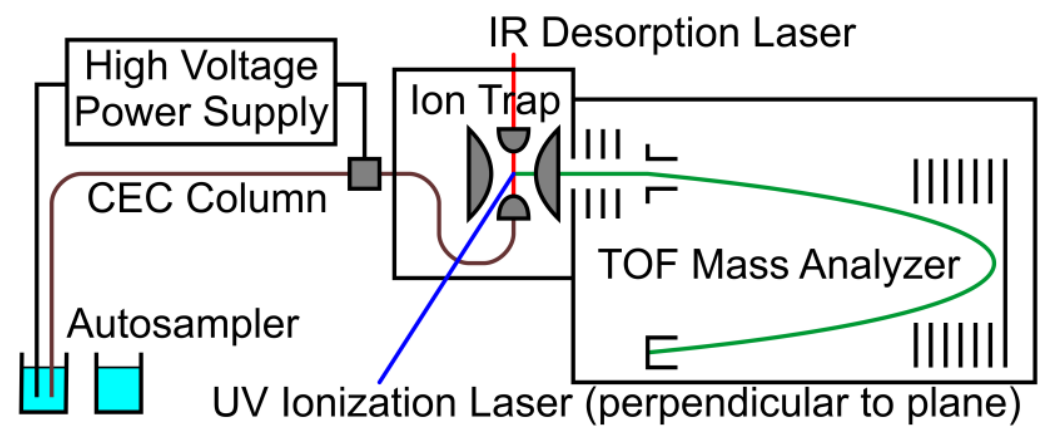

Development of a novel instrument for the hyphenation of capillary electrochromatography with ion-trap accumulation time-of-flight mass spectrometry via a temporally and spatially separated laser desorption laser ionization interface. 